\title{
Factor Analysis Reveals Student Thinking using the Mechanics Reasoning Inventory
}

\author{
Sunbok Lee ${ }^{1}$ \\ sunbok@mit.edu \\ Alex Kimn ${ }^{1}$ \\ akimn@mit.edu
}

\author{
Zhongzhou Chen ${ }^{2}$ \\ Zhongzhou.Chen@ucf.edu \\ Andrew Paul ${ }^{3}$ \\ pawla@uwplatt.edu
}

\author{
David Pritchard ${ }^{1}$ \\ dpritch@mit.edu
}

\begin{abstract}
The Mechanics Reasoning Inventory (MRI) [1] is an assessment instrument specifically designed to assess strategic reasoning skills involving core concepts in introductory Newtonian mechanics. Being an assessment of higher order thinking (as opposed to declarative or rulebased procedural thinking), it is necessary to check whether or not the mental constructs underlying actual student responses correlate with the authors' domain classification, which is the subject of this paper. The instrument consists of three types of problems: whether momentum or energy is conserved in a given situation and why, (partly inspired by the paired what/why questions in Lawson's Classroom Test of Scientific Reasoning), application of Newton's 2nd and 3rd law, and decomposing problems into parts (inspired by Van Domelen [2]'s Problem Decomposition Diagnostic). It has been administered 183 times in two MIT courses since 2009. Exploratory Factor Analysis (EFA) revealed that each Lawson pair of questions should be considered as one item, after which it identified four factors among the 21 questions that correspond reasonably well with the intended physics topics, and a fifth factor correlated with the concept of circular motion, a difficult topic for students (even though not viewed as a core principle by the designers). We discuss why 6 of the items classified under factors that differed from the expert assignments. There was no strong indication that the students answered each of different problem types similarly, which is a hallmark of students using novice heuristics rather than reasoning based on physical principles to answer the questions.
\end{abstract}

Author Keywords

mechanical reasoning inventory; exploratory factor analysis

ACM Classification Keywords

I.2.4 Knowledge Representation Formalisms and Methods

Permission to make digital or hard copies of part or all of this work for personal or classroom use is granted without fee provided that copies are not made or distributed for profit or commercial advantage and that copies bear this notice and the full citation on the first page. Copyrights for third-party components of this work must be honored. For all other uses, contact the owner/author(s). Copyright is held by the author/owner(s).

L@S 2017, April 20-21, 2017, Cambridge, MA, USA

ACM ISBN 978-1-4503-4450-0/17/04.

DOI: http://dx.doi.org/10.1145/3051457.3053984

\section{INTRODUCTION}

As the emphases of both online education and this conference turn more toward learning, and as the goals of learning turn toward "higher" level cognitive skills such as critical thinking and problem solving [p21.org], it is important to develop assessments that can be deployed at scale to measure whether these goals have been met. This paper analyzes a large data set of students' responses to an assessment designed to probe strategic knowledge of introductory Newtonian Mechanics; the Mechanics Reasoning Inventory (MRI) [1]. While experts can perform cognitive task analysis to find the knowledge components necessary in a simple domain like arithmetic, the mental constructs that underlie something like strategic thinking are harder to determine. (Note: In this paper we use mental constructs, familiar from psychometrics, which is somewhat broader than a knowledge component.) Mental constructs are often determined by observing people actually doing the target task - for example, Tuminaro and Redish [8] found students applying several different “epistemic games” while solving physics problems. Observation is important because several lines of research show that novice students do not use the same mental constructs as experts when solving the same problem $[3,4]$

The MRI was designed from an expert's point of view with the objective of measuring students' ability to determine and explain which core physics principle applies in a given problem situation. In light of the previous concerns, we must therefore address two research questions:

1. What mental constructs do the students actually use to answer the questions?

2. Are these the same constructs that the assessment authors' designed the assessment to measure?

These questions are of concern in: psychometrics, data mining, machine learning, and physics education research. We discuss below why we selected Exploratory Factor Analysis (EFA) for this study, and how large an educational data set is required to make it work. Of pedagogical interest, our study demonstrates one route to developing assessments of higher level mental constructs- in this case of strategic knowledge (as opposed to the more usually assessed declarative or procedural knowledge). 


\section{METHODS}

\section{Data Collection and Selection}

WE analyze data only from the latest version (MRI.v5), which was used as pre or post test in two MIT remedial physics courses: 8.IAP and 8.011. The former is a threeweek remedial course administered to students who had earned a D in their introductory physics course (8.01) the previous semester, while the latter was a semester long remedial course for those who failed 8.01.

Altogether 185 test instances were collected from 8 different classes (one class used MRI for both pre and post test), all taught by author DEP. Two of the 8.011 classes had pre-test data, for a total of 60 administrations, and a different two had post-test data, for a total of 49 post test instances.

\section{Applying exploratory factor analysis to the MRI data}

Basically, identifying the underlying mental constructs of students requires dimension reduction techniques that reduce the number of variables necessary to explain the data below the number of items. Among many dimension reduction techniques for item response data, we used EFA to identify the underlying factor structure of the MRI. In EFA, the covariance structures of observed variables are assumed to be explained by a small number of underlying latent variables, which is usually called factors. EFA has been widely used to explore underlying factor structures of educational tests and psychological assessments, and has some advantages over other methods. First, EFA is the model that reflects our theoretical assumption about the relationship between underlying mental constructs and observed items responses because factors are formulated as the causes or predictors of correlated item responses. Second, EFA explicitly models measurement errors by separating common and unique sources of variance in item responses. It is known that ignoring measurement errors can cause bias [5]. We used the Mplus software package [7] to handle binary responses (right or wrong) from the MRI.

\section{Initial factor analysis and data processing}

In our initial EFA analysis, we treated each question in the MRI as an item. This approach identified factors that consisted largely of the statement/justification pairs in the first and last section of the instrument, which is an unsurprising but uninformative result. Furthermore, treating the paired problems as separate items clearly violates the premises of FA that the solution to each item is independent. Therefore, each statement/justification pair was treated as one item, judging the combined item correct only when both statement and justification are answered correctly (using a less strict criteria resulted in nonconverging FA result). Furthermore, author AP identified from student interviews that the wording of problems 5 and 6 caused some students to incorrectly interpret the problems. Therefore, those two problems were discarded from the FA analysis. The resulting data now comprise 14 items, 5 of them consisting of a pair of questions.

\section{Determining the Number of Factors}

One of the key decisions in EFA is to decide the number of meaningful factors. Several criteria have been proposed in literature [5]. First, factors with eigenvalues greater than 1 can be considered to be meaningful factors. Second, factors can also be retained based on the scree plot in which the eigenvalues are plotted against their corresponding factors. In the scree plot, the number of meaningful factors can be determined by the number of eigenvalues above the breakpoint of the scree plot, or the point between the steep slope and the leveling-off. Third, the optimal number of factors can be determined based on the model comparison.

\begin{tabular}{|c|c|c|c|c|c|c|c|c|c|c|c|c|c|c|}
\hline & I1-2 & I7 & I16-17 & I18-19 & I20-21 & I3-4 & I8 & I13 & I11 & I15 & I9 & $\mathrm{I} 10$ & I14 & I12 \\
\hline Cluster & 1 & 1 & 1 & 1 & 1 & 2 & 2 & 3 & 3 & 3 & 4 & 4 & 5 & 5 \\
\hline F1 & $0.92 *$ & $0.73 *$ & $0.57 *$ & $0.47 *$ & $0.45^{*}$ & -0.05 & $0.35^{*}$ & -0.22 & 0.05 & 0.09 & 0.00 & 0.05 & 0.03 & 0.02 \\
\hline F2 & -0.05 & 0.06 & 0.20 & $0.41 *$ & -0.07 & $0.90 *$ & $0.37 *$ & 0.01 & -0.22 & 0.23 & 0.04 & -0.03 & 0.00 & -0.08 \\
\hline F3 & 0.03 & 0.03 & 0.06 & -0.06 & 0.02 & 0.16 & -0.14 & $1.10 *$ & $0.78 *$ & $0.47 *$ & 0.03 & -0.03 & $0.56^{*}$ & 0.20 \\
\hline F4 & 0.01 & 0.20 & -0.07 & -0.06 & 0.15 & 0.04 & 4 & 0.04 & 0.00 & -0 & $0.96 *$ & 0.53 & -0.01 & 0.12 \\
\hline F5 & $-0.55 *$ & 0.02 & 0.04 & 0.19 & 0.28 & 0.01 & -0.23 & -0.02 & 0.08 & -0.13 & -0.04 & 0.14 & $0.74 *$ & 0.36 \\
\hline Disc & 0.99 & 1.50 & 0.99 & 088 & 1.18 & 0.43 & 0.54 & 1.21 & 120 & 0.73 & 0.86 & 0.81 & 1.39 & 0.80 \\
\hline Diff & -0.40 & -1.01 & -0.42 & 1.98 & 1.83 & -1.82 & 2.22 & -1.57 & -1.02 & -1.70 & 0.44 & 2.46 & -1.35 & -3.26 \\
\hline
\end{tabular}

Note. * indicates that a factor loading is statistically significantly different from zero at $5 \%$ level of significance. Bold fonts are used to represent factor loadings greater than 0.3. The numbers in the row named cluster indicate the cluster identified by the hierarchical cluster analysis. F1-F5 represent factor 1 through 5.

Table 2. Factor loadings from EFA. 
Mplus provides us a series of tests for comparing the Chisquare model fit indices between two consecutive models.

\section{RESULTS}

In this study, we adopt the criteria that factors with eigenvalues greater than 1 can be considered to be meaningful factors. As shown in Figure 1, this eigenvalue criterion supports a 5-factors model.

A simple loading structure for the five-factor model is produced by the Mplus software and presented in Table 2. We adopted three different criteria to interpret this loading matrix. First, the relationship between an observed variable and a factor can be considered to be meaningful when the corresponding loading is greater than .3. Second, items can be clustered to give maximum interpretability using the hierarchical cluster analysis [6]. The row named cluster in Table 2 represents the clusters obtained from the hierarchical cluster analysis using $\mathrm{R}$ package psych. Third, Mplus provides the results of statistical tests for checking whether each loading is statistically significantly different from zero. All three criteria are displayed in Table 2 (see Note in Table 2).

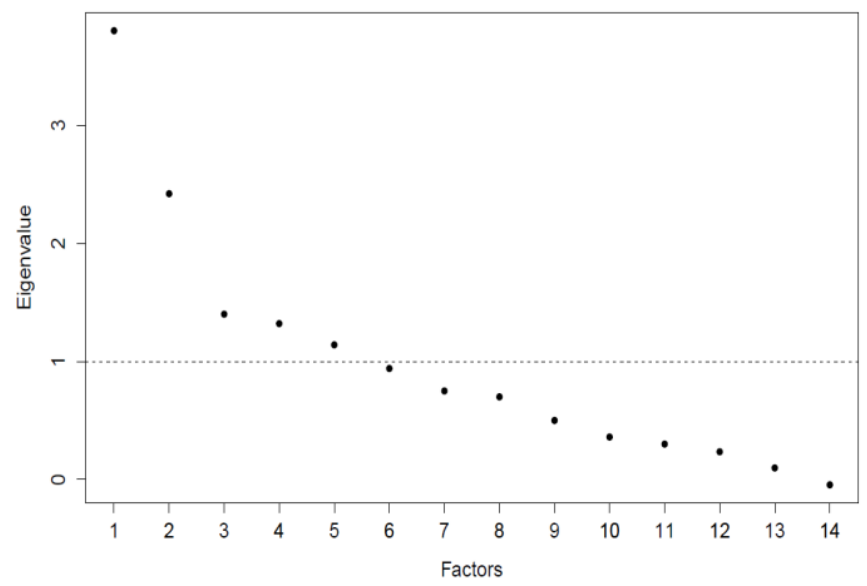

Figure 1.The scree plot representing eigenvalues against factor number. The dotted line represents eigenvalue equal to 1 . DISCUSSION AND FUTURE DIRECTIONS

If a fine-grained assessment is to be used to guide instruction - and especially to guide remediation of a particular student's weak points - it must provide ratings aligned with how the target audience organizes its domain knowledge. For example, if a student approaches physics problems using a plug and chug method (which is agnostic to physical concepts) remediation for that student needs to start at a very basic level. But if statistical errors or extra difficulties for plug and chug resulted in more mistakes on those questions dealing with mechanical energy, then analysis based on the expert domain map will guide us to clarifying the concept of mechanical energy for that student - something that is foreign to that student's thinking and won't address that student's fundamental misunderstanding.
Unlike many concept inventories that focus on probing one or two topics at a time, the MRI involves multiple topics from introductory mechanics. It is intended to assess four different concepts (Newton's Law \#2, Newton's Law \#3, Momentum, and Energy) in three different problem formats. Therefore, factor analysis is particularly informative for MRI, as it can illuminate our research questions:

1. What mental constructs do the students actually use to answer the questions?

2. Are these the same constructs that the assessment authors' designed the assessment to measure?

Factor analysis might also reveal clusters corresponding to different problem formats - an indication that students may be using heuristic methods to answer questions on the MRI. And by comparing with final exam scores, we might infer the importance of the strategic knowledge required for the MIR for overall problem solving ability.

\section{Interpretation of the five-factor model}

The outstanding result of the analysis (see Table 1 and 2) is that five significant factors were discovered whereas the instrument was designed with 4 . Examining Table 2 shows that factor 4 is measured heavily only by items 9 and 10, and that these items load far more heavily on factor 4 than on the other factors. These questions are unique wrt the MRI because both involve "circular motion" in addition to (the designed) concepts of energy and momentum. The fact that these items have very light loadings on other factors indicates that students find that understanding circular motion is the most significant concept in the problem in spite of the presence of other important concepts (to the experts). Both factors 3 and 5 belong to the middle section involving Newton's laws. Interestingly, the two factors do not correspond to second law vs. third law as intended. Upon a closer look at the problems, we noticed that in problems 11 and 13, the problem body shares a common structure of "force on A from B and force on A from C are:" whereas the correct answer is "newton's second law applied to A.” Problem 15 asks which pair of forces remain equal when the safe is accelerating, and the correct choice, "force from person on safe and force from safe on person", has a similar symmetric grammatical structure emphasizing the word which is key to the correct answer. Such grammatical cues towards the correct answer are absent from problems 12 and 14 . Therefore, a plausible interpretation of the factors may be that a significant fraction of students employed (novice) heuristic methods based on verbal analysis to solve the problem. We plan to rewrite these items in a way that such heuristic methods will not work.

Granting the reclassification of the circular motion questions and the two of the 5 Newton's Law questions, the 
factor analysis and the expert assignments differ only for the two question pairs 16-17 and 17-18. The reasons for this are not clear to us. Although 16-17 might be classified as kinematics, there seems to be no reason to think that either this problem or 18-19 is a momentum problem. This suggests that perhaps factor 1 is not purely momentum. We plan to investigate this further.

In summary, we regard the application of EFA to the MRI.v5 as generally successful. It clearly revealed that the two questions on circular motion were mis-classified by the experts due to student difficulties with the concept of circular motion. This left only 6 of the 21 questions where the expert classification disagreed with the factors revealed by the EFA. We hope that careful investigation of the particular patterns of wrong answers will give insight into what is going on in the minds of the students and allow us to revise the items to get clearer factor loadings. Beyond that, we can write and analyze new questions or seek to interview students on these questions.

One final qualification: the relative consistency between expert classification and EFA is somewhat unusual in physics. However, it might be due to the fact that most conceptual inventories focus on one general topic and one problem type, whereas the MRI spans multiple topics and problem formats. College students are in the early stages of the novice to expert transition process, and therefore they may have learned the expert categorization on the topic level (helpful here), but not at the subtopic level required for most concept tests.

\section{Implications for Future Development}

The FA results imply a certain level of validity of the MRI. Notably, the second and third sections of the instrument seem to be each testing its own underlying skill, whereas the first section is more related to students' topical understanding.

The results also provide important information for the continuous improvement of the instrument. For example, the wording of problems $11-15$ needs to be improved to prevent the use of heuristic methods. In fact, interviews conducted at University of Wisconsin Platteville suggested that many students believe the pair of forces given weren't equal to each other. The absence of this option in the current version could have driven students towards heuristic methods.
It would also be wise to add some energy and momentum questions involving the situation in the middle section. Finding a way to collect more data more quickly would also be helpful.

\section{Authors' Affiliation}

1= Massachusetts Institute of Technology, Cambridge MA; 2=University of Central Florida, Orlando FL; 3=University of Wisconsin, Platteville WI.

\section{REFERENCE}

1. Andrew Pawl, Analia Barrantes, Carolin Cardamone, Saif Rayyan, David E. Pritchard, N. Sanjay Rebello, Paula V. Engelhardt, and Chandralekha Singh. 2011. Development of a mechanics reasoning inventory. In Physics Education Research Conference, 287-290. https://doi.org/10.1063/1.3680051

2. David Van Domelen. 2000. The development of the problem decomposition diagnostic.

3. Michelene T.H. Chi, Paul J Feltovich, and Robert Glaser. 1981. Categorization and representation of physics problems by experts and novices. Cognitive Science 5: 121-152. Retrieved from papers2://publication/uuid/66AFC0DE-6DB6-42768C3A-C90185B1064F

4. Jonathan Tuminaro and Edward Redish. 2007. Elements of a cognitive model of physics problem solving: Epistemic games. Physical Review Special Topics - Physics Education Research 3, 2: 1-22. https://doi.org/10.1103/PhysRevSTPER.3.020101

5. Jum C Nunnally, Ira H Bernstein, and Jos M F ten Berge. 1967. Psychometric theory. JSTOR.

6. William Revelle. 1979. HIERARCHICAL CLUSTER ANALYSIS AND TIHE INTERNAL STRUCTURE O F TESTS. Multivariate Behavwral Research 14: 5774.

7. Linda K Muthén and Bengt O Muthén. 2010. Mplus User's Guide: Statistical Analysis with Latent Variables: User'ss Guide. Muthen \& Muthen.

8. Jonathan Tuminaro and Edward Redish. 2007. Elements of a cognitive model of physics problem solving: Epistemic games. Physical Review Special Topics - Physics Education Research 3, 2: 1-22. https://doi.org/10.1103/PhysRevSTPER.3.020101 